\title{
The effect of albendazole treatment on non-seizure outcomes in patients with symptomatic neurocysticercosis
}

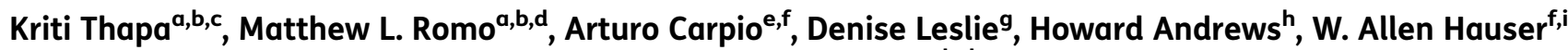 \\ and Elizabeth A. Kelvin ${ }^{a, b, d, *}$
}

\begin{abstract}
${ }^{a}$ Epidemiology \& Biostatistics Program, School of Urban Public Health at Hunter College, City University of New York, 2180 Third Avenue, New York, NY 10035, USA; ${ }^{b}$ CUNY Graduate School of Public Health and Health Policy, City University of New York, 55 West $125^{\text {th }}$ Street, New York, NY 10027, USA; ' University of Rochester School of Medicine and Dentistry, 601 Elmwood Avenue, Rochester, NY 14642, USA; ${ }^{d}$ CUNY Institute for Implementation Science in Population Health, City University of New York, 55 West $125^{\text {th }}$ Street, New

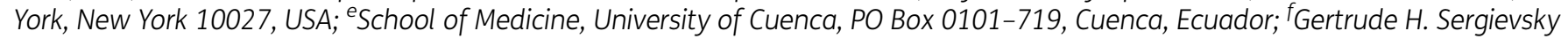

Center, College of Physicians and Surgeons, Columbia University, 622 West 168th Street, New York, New York 10032, USA; ${ }^{9}$ MRI

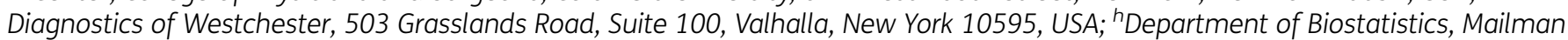
School of Public Health, Columbia University, 722 West 168th Street, New York, New York 10032, USA; 'Department of Epidemiology, Mailman School of Public Health, Columbia University, New York, New York, USA

*Corresponding author: Tel: +646-364-9610; E-mail: Elizabeth.Kelvin@sph.cuny.edu
\end{abstract}

Received 20 September 2017; revised 29 December 2017; editorial decision 19 February 2018; accepted 19 February 2018

Background: There is little information about the impact of anthelminthic treatment on clinical symptoms other than seizures in neurocysticercosis (NC). We investigated the effect of albendazole on non-seizure symptoms experienced by patients with NC.

\begin{abstract}
Methods: Data are from a randomized controlled trial comparing albendazole plus prednisone with placebo plus prednisone for treatment of NC among 173 patients with active or transitional NC cysts and new-onset symptoms. We performed negative binomial regression to examine the number of follow-up visits when a symptom was reported, logistic regression to examine the probability of experiencing the symptom and Cox proportional hazards models to examine the time to first reporting the symptom.

Results: Eighty-five percent of patients reported at least one non-seizure symptom at baseline. Those treated with albendazole had significantly lower odds of memory loss and/or confusion during months 1-24 (odds ratio [OR] 0.42, $\mathrm{p}=0.037$ ) and significantly increased odds of anxiety and/or depression during months $1-12$ (OR 1.87, $\mathrm{p}=0.049$ ). No treatment difference existed in experiencing symptoms in general or in experiencing headaches, limb weakness or gait disturbances, vomiting, nausea and/or stomach pain or visual disturbances over the follow-up period.

Conclusions: While the prevalence of non-seizure symptoms was high, albendazole treatment was associated with only two significant differences in the non-seizure symptoms over follow-up. Further research is needed to identify strategies to reduce the long-term symptom burden in patients with NC.
\end{abstract}

Keywords: Albendazole, Cestode, Cysticercosis, Helminth, Neurocysticercosis, Parasitic, Taenia solium

\section{Introduction}

Neurocysticercosis (NC) is an infection of the central nervous system (CNS) with the larval stage of the tapeworm Taenia solium. It is the most common helminthic infection of the CNS and is endemic in most countries of Latin America, Asia and sub-Saharan Africa. ${ }^{1,2} \mathrm{NC}$ is a serious public health problem in many low- and middle-income nations as well as in high-income nations with substantial immigration from endemic countries. ${ }^{3}$ Designated as an emerging infection by the US Centers for Disease Control and Prevention (CDC), ${ }^{4} \mathrm{NC}$ accounts for more than 1000 diagnosed cases per year in the USA. ${ }^{5}$

The larval form of the parasite has an affinity for CNS tissue in humans and is a major cause of neurological morbidity and mortality in endemic countries. ${ }^{6} \mathrm{NC}$ is possibly the most heterogeneous infection of the CNS, with a wide variety of clinical manifestations, and may be fatal or completely asymptomatic. While seizures are often reported to be the most common symp-

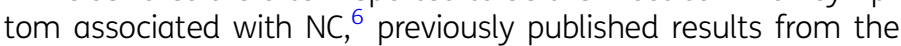

(c) The Author(s) 2018. Published by Oxford University Press on behalf of Royal Society of Tropical Medicine and Hygiene. All rights reserved. For permissions, please e-mail: journals.permissions@oup.com. 
data for this paper from the Ecuadorian Neurocysticercosis Group (ENG) found that the most common presenting symptom was headache, followed by seizures; gastrointestinal (GI) symptoms, including nausea, abdominal pain and/or vomiting; equilibrium and/or gait disturbances; vision problems, including double vision and/or other visual symptoms; depression and/or anxiety and memory loss and/or confusion.?

Randomized controlled trials (RCTs) have found treatment with anthelmintic drugs such as albendazole to be effective in causing the death of the parasite and resolving active cysts located in the parenchyma and therefore this treatment has become standard care for NC. ${ }^{8}$ However, there is little information about the impact of albendazole on clinical symptoms associated with the disease. ${ }^{8-12}$ One meta-analysis examining the treatment of parenchymal solitary cysticercus granuloma with albendazole found improved rates of seizure freedom and more rapid resolution of the granuloma. ${ }^{13}$ Another meta-analysis of trials looking at the impact of anthelmintic treatment on NC-related seizures suggested that the treatment is 'probably effective' in decreasing long-term seizure frequency in patients with parenchymal NC. ${ }^{8}$ Two studies, including one from the ENG trial, found a lower number of seizures with generalization following treatment with albendazole compared with placebo, but no difference in the number of seizures overall or the number of seizures without generalization. ${ }^{14,15}$ However, a differential impact of albendazole on generalized vs partial seizures does not seem to be biologically plausible given our current understanding of the causes of seizures in patients with NC, and the explanation for this finding is not yet known. ${ }^{14}$ The lack of association of albendazole with seizures without generalization could be due to small sample sizes and low statistical power.

There is almost no information about the potential impact of anthelmintic treatment on non-seizure symptoms. The ENG trial looked at differences by treatment arm for a few common or severe symptoms (headache, gastrointestinal symptoms and intracranial hypertension) up to 1 month post-treatment and found no significant difference between those treated with albendazole vs placebo. ${ }^{7}$ Another study found that albendazole was associated with an increased proportion of patients with encephalopathy and hospital readmission among those treated with albendazole plus steroids vs placebo at 3 months (30\% vs $10 \% ; \chi^{2}$ $\mathrm{p}<0.01)$ and 1 y $\left(1.35 \%\right.$ vs $\left.0.66 \% ; \chi^{2} \mathrm{p}=0.05\right) .{ }^{16}$ However, the numbers for these comparisons are small (at $1 \mathrm{y}$, only three patients experienced encephalopathy, two in the albendazole group), the study was not double-blinded and significance testing was done with $\chi^{2}$ tests rather than exact tests, which might be misleading. A better understanding of the clinical impact of albendazole treatment for $\mathrm{NC}$ will enable clinicians to make better treatment decisions with their patients. Therefore the aim of this study was to evaluate the impact of albendazole treatment (plus steroids) vs placebo (plus steroids) for NC on non-seizure symptoms over a 2-year follow-up period using data from the ENG trial.

\section{Materials and methods}

\section{Study design}

The ENG RCT, from which the data for this study come, has been previously described (ClinicalTrials.gov identifier: NCT00283699)., ${ }^{74,17-20}$
Briefly, the study was a randomized, double-blind, placebocontrolled trial conducted among 173 patients with newly diagnosed symptomatic NC seeking care in one of six hospitals in Ecuador. Eligible participants were those who presented with new onset of NC-associated symptoms within the past 2 months and had active and/or transitional NC cysts present on computed tomography (CT) or magnetic resonance imaging (MRI). Patients with calcified cysts who also had either active and/or transitional cysts were also eligible for the study, but those with only calcified cysts were not eligible. In addition, those who were pregnant or had active tuberculosis, syphilis, papilledema, ocular cysticercosis, active ulcers or any progressive or life-threatening disorder were ineligible. Patients who had received treatment for NC during the past year or who had undergone systemic treatment with steroids within $30 \mathrm{~d}$ of presentation were also ineligible for this study; also, midway through the study, patients who already had a ventricular shunt were excluded due to safety concerns.

Participants included in this study received albendazole $400 \mathrm{mg}$ $(n=86)$ or placebo $(n=87)$, given orally (directly observed) every $12 \mathrm{~h}$ for $8 \mathrm{~d}$. All participants received prednisone $75 \mathrm{mg}$ daily for $8 \mathrm{~d}$, which was then tapered over 2 weeks. For patients weighing $<50 \mathrm{~kg}$, the doses of albendazole and prednisone were reduced in a standardized manner. Patients were also given symptomatic treatment, described elsewhere. ${ }^{7}$ Patients were followed for 24 months, with follow-up visits occurring at months $1,2,3,4,5,6$, 9, 12, 15, 18 and 24.

\section{Measures}

At baseline and each follow-up visit, symptoms were assessed by a nurse using a standardized questionnaire in which patients were asked to report the presence, frequency, and severity of any symptoms they had experienced in the past 2 months (at baseline) or since the previous interview (at follow-up) through the reading of a symptom checklist, after which participants were asked if they had experienced any other symptoms that were not included in the list. Symptoms on the list were thought to be potentially related to the NC or to treatment for NC and therefore we wanted a baseline assessment to describe the symptomatic profile of NC patients and to track changes over time.

For the present analysis, the two primary outcomes examined were experiencing any symptom over the follow-up (including seizures) or any non-seizure symptom. In addition, we looked at the six most common symptoms reported at baseline for which we might have sufficient statistical power to find a difference and which have been shown in the past to be associated with NC, including headaches, ${ }^{21,22}$ limb weakness ${ }^{23,24}$ or gait disturbances, ${ }^{25,26}$ anxiety and/or depression, ${ }^{27-29}$ problems with vision (double vision and/or other vision difficulties), ${ }^{21,22}$ GI symptoms (vomiting, nausea and/or stomach pain) $22,26,30$ and memory loss and/or confusion. ${ }^{21,28,31}$

Time periods for symptom endpoints were categorized into months $1-12$, months $13-24$ and months $1-24$, as has been done in previous studies. ${ }^{14,15}$ We looked at each symptom in three ways: (1) as a dichotomous indicator for having experienced the symptom at any time during the follow-up period being examined, (2) as a count of the number of follow-up visits (months 1, 2, 3, 4, 
$5,6,9,12,15,18$ and 24) during which the patient reported having experienced the symptom at least once since their previous followup visit and (3) as the time to first experiencing the symptom following treatment.

\section{Statistical analysis}

We describe the symptoms experienced by the NC patients at baseline and over the follow-up. Pearson's $\chi^{2}$ or Fisher's exact (when expected cell counts were $<5$ ) tests were used to assess the statistical significance of the association between having a symptom at baseline and having it during each follow-up period.

Data were analysed in an intention-to-treat approach to take advantage of the prevention of confounding from the randomization. Logistic regression (presence of the symptom during follow-up), negative binomial regression (number of follow-up visits at which the patient reported having the symptom), and Cox proportional hazards (time from treatment to first experience of the symptom during follow-up) models were used to determine the association between treatment and the odds, frequency and hazards of having each symptom examined, respectively, over time.

Overall, at 24 months, $59.1 \%(52 / 88)$ of patients randomized to albendazole and $58.9 \%$ (53/90) of patients randomized to placebo had at least some missing data either due to loss to follow-up or to missing a study visit; those with missing data were excluded from complete case analyses for all regression models. In order to ensure that our results were not due to missing data, which can decrease statistical power as well as bias associations, we used multiple imputation to address missing data and reran the logistic regression models with the imputed data. As there is no published literature on predictors of experiencing non-seizure symptoms in $\mathrm{NC}$, the variables used to impute data were those that have been found to predict seizures, ${ }^{14,18}$ as well as some that seemed clinically plausible predictors of each symptom. These variables included participant's age, sex, treatment assignment, total number of cysts at baseline and presence of the symptom at baseline. Using Rubin's procedure, five imputations were performed and the logistic regression models were then rerun with the missing data replaced with the imputed values. ${ }^{32}$ All analyses were performed using SAS 9.3 (SAS Institute, Cary, NC, USA).

\section{Results}

\section{Description of symptoms}

All participants experienced some clinical symptom at baseline, as this was an eligibility criterion. Overall, 85\% (147/173) of patients reported at least one non-seizure symptom at baseline. At baseline, the most commonly occurring non-seizure symptoms were as follows: headaches, 72\% (124/173); GI symptoms, 34\% (58/173); limb weakness and/or gait problems, 26\% (44/171); anxiety and/or depression, $17 \%$ (30/173); double vision and/or other vision difficulties, 17\% (30/172); memory loss and/or confusion, 16\% (27/ 171). Results regarding seizures for this study have been described elsewhere. ${ }^{14}$ By the end of the 24-month follow-up period, all patients (104/104 with complete follow-up) had experienced some symptoms during follow-up and 88\% (91/104) had experienced at least one non-seizure symptom. There was no significant association between having experienced any symptoms at baseline and experiencing the same symptom during follow-up; however, with the exception of headaches, there was a pattern for a higher proportion of those who had not reported the symptom at baseline to report it during follow-up compared with those who had reported the symptom at baseline (Table 1).

\section{Effect of albendazole treatment on symptom frequency}

In the negative binomial regression models, there were no significant treatment effects on the frequency of any symptoms (Table 2).

\section{Effect of albendazole on the odds of having a symptom over follow-up}

In the logistic regression models, those who received albendazole treatment had 0.42 times lower odds of memory loss and/or confusion over the 24-month follow-up compared with those who received placebo (95\% confidence interval [CI] 0.19 to 0.95). The association remained in the model with missing data imputed (months 1-24 OR 0.47 [95\% CI 0.22 to 0.99]). In addition, those treated with albendazole had 1.87 times greater odds of anxiety and/or depression during months 1-12 follow-up (OR 1.87 [95\% CI 1.01 to 3.53]) compared with those in the placebo group in the model with imputed data. There were no other significant associations between treatment and odds of having a symptom during follow-up in the logistic regression models (Table 3).

\section{Effect of albendazole on time to symptom over follow-up}

There were no significant associations between treatment and time to symptom over follow-up in any of the Cox proportional hazards models (Table 4).

\section{Discussion}

There is almost no research describing the clinical symptoms experienced by those presenting with NC. The one exception is seizure, which has been investigated in a few studies, ${ }^{33}$ but those infected with NC can present with a number of different symptoms. ${ }^{6}$ In this study, we describe these symptoms in a cohort of 173 patients with new-onset symptoms and NC diagnosed with CT or MRI. We found that headache was the most common symptom experienced, followed by seizure, which is not consistent with the literature, which describes seizure as the most common presenting symptom. ${ }^{6}$ Symptoms experienced likely depend on the location of the parasites: seizure is the most frequent symptom in the parenchymal forms of NC, but headache is most frequent in the extraparenchymal forms. ${ }^{3}$ In addition, we found that the majority of patients with NC experienced continued or new-onset symptoms over follow-up, despite treatment with steroids (which all those in this study received) and treatment for the symptoms reported when appropriate. Furthermore, the probability of experiencing a specific non-seizure symptom during follow-up was, in most cases, unrelated to whether or not that symptom was reported at baseline. 
Table 1. Symptoms at baseline (2 months prior to enrolment) and over the 24 months of follow-up among randomized patients with complete data only

\begin{tabular}{|c|c|c|c|c|c|c|c|c|c|c|c|c|c|}
\hline \multirow[t]{2}{*}{ Symptom at baseline } & \multirow[t]{2}{*}{$\begin{array}{l}\text { Baseline, } \\
\text { n (\%) }\end{array}$} & \multicolumn{3}{|c|}{ Baseline to 6-months follow-up } & \multicolumn{3}{|c|}{$\begin{array}{l}\text { Baseline to } 12 \text {-months follow- } \\
\text { up }\end{array}$} & \multicolumn{3}{|c|}{$\begin{array}{l}\text { Baseline to } 18 \text {-months follow- } \\
\text { up }\end{array}$} & \multicolumn{3}{|c|}{ Baseline to 24-months follow-up } \\
\hline & & Yes, n (\%) & No, n (\%) & p-Value & Yes, n (\%) & $\begin{array}{l}\text { No, } n \\
(\%)\end{array}$ & $\mathrm{p}$-Value & Yes, n (\%) & $\begin{array}{l}\text { No, n } \\
(\%)\end{array}$ & p-value & Yes, n (\%) & No, n (\%) & $\mathrm{p}$-Value \\
\hline Any symptom & & & & NA & & & NA & & & NA & & & NA \\
\hline Yes & $173(100.0)$ & $165(100.0)$ & $0(0.0)$ & - & $161(100.0)$ & $0(0.0)$ & - & $133(100.0)$ & $0(0.0)$ & - & $104(100.0)$ & $0(0.0)$ & - \\
\hline No & $0(0.0)$ & $0(0.0)$ & $0(0.0)$ & & $0(0.0)$ & $0(0.0)$ & & $0(0.0)$ & $0(0.0)$ & & $0(0.0)$ & $0(0.0)$ & \\
\hline $\begin{array}{l}\text { Any non-seizure } \\
\text { symptoms }\end{array}$ & & & & NA & & & NA & & & NA & & & NA \\
\hline Yes & $147(85.0)$ & $141(100.0)$ & $0(0.0)$ & & $138(100.0)$ & $0(0.0)$ & & $115(100.0)$ & $0(0.0)$ & - & $91(100.0)$ & $0(0.0)$ & - \\
\hline No & $26(15.0)$ & $24(100.0)$ & $0(0.0)$ & & $23(100.0)$ & $0(0.0)$ & & $18(100.0)$ & $0(0.0)$ & & $13(100.0)$ & $0(0.0)$ & \\
\hline \multicolumn{14}{|l|}{ Headaches } \\
\hline Yes & $124(71.7)$ & 109 (92.4) & $9(7.6)$ & $0.730^{\mathrm{b}}$ & $109(94.0)$ & $7(6.0)$ & $1.000^{\mathrm{b}}$ & $92(96.8)$ & $3(3.2)$ & $0.624^{b}$ & $71(100.0)$ & $0(0.0)$ & $0.094^{b}$ \\
\hline No & $49(28.3)$ & $45(95.7)$ & $2(4.3)$ & & $43(95.6)$ & $2(4.4)$ & & $36(94.7)$ & $2(5.3)$ & & $30(93.8)$ & $2(6.3)$ & \\
\hline \multicolumn{14}{|c|}{ Limb weakness/gait disturbance } \\
\hline Yes & $44(25.7)$ & $16(37.2)$ & $27(62.8)$ & $0.485^{a}$ & $19(44.2)$ & $24(55.8)$ & $0.515^{a}$ & $18(47.4)$ & $20(52.6)$ & $0.316^{a}$ & $16(51.6)$ & $15(48.4)$ & $0.703^{a}$ \\
\hline No & $127(74.3)$ & $52(43.3)$ & $68(56.7)$ & & $58(50.0)$ & $58(50.0)$ & & $53(57.0)$ & $40(43.0)$ & & $39(55.7)$ & $31(44.3)$ & \\
\hline \multicolumn{14}{|l|}{ Anxiety/depression } \\
\hline Yes & $30(17.3)$ & $14(48.3)$ & $15(51.7)$ & $0.810^{a}$ & $16(55.2)$ & $13(44.8)$ & $0.813^{\mathrm{a}}$ & $16(61.5)$ & $10(38.5)$ & $0.643^{a}$ & $16(64.0)$ & $9(36.01)$ & $0.384^{\mathrm{a}}$ \\
\hline No & $143(82.7)$ & $69(50.7)$ & $67(49.3)$ & & $76(57.6)$ & $56(42.4)$ & & $71(66.4)$ & $36(33.6)$ & & $57(73.1)$ & $21(26.9)$ & \\
\hline \multicolumn{14}{|c|}{ Vision problems (double vision, other vision difficulties) } \\
\hline Yes & $30(17.4)$ & $6(20.7)$ & $23(79.3)$ & $0.856^{a}$ & $6(21.4)$ & $22(78.6)$ & $0.386^{\mathrm{a}}$ & $6(21.4)$ & $22(78.6)$ & $0.386^{\mathrm{a}}$ & $6(23.1)$ & 20 (76.9) & $0.216^{\mathrm{a}}$ \\
\hline No & $142(82.6)$ & $30(22.2)$ & $105(77.8)$ & & 39 (29.6) & $93(70.5)$ & & $39(29.6)$ & $93(70.4)$ & & $38(35.9)$ & $68(64.2)$ & \\
\hline \multicolumn{14}{|c|}{ GI symptoms (vomiting, nausea, stomach pain) } \\
\hline Yes & $58(33.5)$ & $24(42.9)$ & $32(57.1)$ & $0.482^{a}$ & $26(46.4)$ & $30(53.6)$ & $0.157^{\mathrm{a}}$ & $28(52.8)$ & $25(47.7)$ & $0.209^{a}$ & $28(68.2)$ & $13(31.7)$ & $0.772^{\mathrm{a}}$ \\
\hline No & $115(66.5)$ & $53(48.6)$ & $56(51.4)$ & & $61(58.1)$ & $44(41.9)$ & & $51(63.8)$ & $29(36.3)$ & & $44(70.1)$ & $18(29.0)$ & \\
\hline \multicolumn{14}{|l|}{ Memory loss/confusion } \\
\hline Yes & $27(15.8)$ & $6(24.0)$ & $19(76.0)$ & $0.473^{a}$ & $7(29.2)$ & $17(70.8)$ & $0.419^{a}$ & $6(27.73)$ & $16(72.7)$ & $0.443^{a}$ & $6(30.0)$ & $14(70.0)$ & $0.432^{a}$ \\
\hline No & $144(84.2)$ & $43(31.2)$ & $95(68.3)$ & & $51(62.2)$ & $84(37.8)$ & & $39(35.8)$ & $70(64.2)$ & & $32(39.5)$ & $49(60.5)$ & \\
\hline
\end{tabular}


Table 2. Negative binomial regression model results evaluating the rate ratio (RR) for the association between albendazole treatment (vs placebo) and the number of times a symptom was reported during follow-up

\begin{tabular}{|c|c|c|c|c|}
\hline & Albendazole & Placebo & RR (95\% CI) & $\mathrm{p}$-Value \\
\hline \multicolumn{5}{|l|}{ Headache } \\
\hline Months 1-12 & $67(n=79)$ & $70(n=82)$ & 0.87 (0.73 to 1.05$)$ & 0.158 \\
\hline Months 13-24 & $31(n=52)$ & $30(n=52)$ & $0.92(0.60$ to 1.40$)$ & 0.701 \\
\hline Months 1-12 & $34(n=79)$ & $36(n=82)$ & 1.03 (0.64 to 1.64$)$ & 0.914 \\
\hline Months 13-24 & $6(n=52)$ & $8(n=52)$ & $0.94(0.29$ to 3.07$)$ & 0.921 \\
\hline Months 1-24 & $28(n=52)$ & $25(n=52)$ & 0.93 (0.55 to 1.57$)$ & 0.792 \\
\hline \multicolumn{5}{|c|}{ Anxiety/depression } \\
\hline Months 1-12 & $46(n=82)$ & $38(n=80)$ & 1.02 (0.66 to 1.58$)$ & 0.939 \\
\hline Months $13-24$ & $2(n=52)$ & $3(n=51)$ & $0.54(0.05$ to 5.45$)$ & 0.600 \\
\hline Months 1-24 & $20(n=52)$ & $16(n=51)$ & 1.11 (0.60 to 2.06$)$ & 0.734 \\
\hline \multicolumn{5}{|l|}{ GI symptoms } \\
\hline Months 1-12 & $35(n=79)$ & $43(n=82)$ & 0.72 (0.47 to 1.10$)$ & 0.124 \\
\hline Months 13-24 & $10(n=52)$ & $9(n=51)$ & $0.86(0.32$ to 2.37$)$ & 0.779 \\
\hline Months 1-24 & $35(n=52)$ & $32(n=51)$ & $0.74(0.48$ to 1.14$)$ & 0.171 \\
\hline \multicolumn{5}{|c|}{ Memory loss/confusion } \\
\hline Months 1-12 & $20(n=79)$ & $33(n=82)$ & 0.69 (0.41 to 1.17$)$ & 0.164 \\
\hline Months 13-24 & $3(n=52)$ & $7(n=51)$ & 0.32 (0.08 to 1.32$)$ & 0.115 \\
\hline Months 1-24 & $15(n=52)$ & $23(n=51)$ & $0.54(0.29$ to 1.01$)$ & 0.054 \\
\hline
\end{tabular}

There is also little research on the impact anthelmintic treatment for NC has on clinical symptoms over long-term follow-up, with only a few studies looking at seizure ${ }^{14-16}$ and one study also examining the frequency of encephalopathy (headache/vomiting/ altered sensorium). ${ }^{16}$ In this study we examined additional clinical symptoms and investigated the association of albendazole to headache and nausea/vomiting separately. We found that there was little difference in the symptoms experienced during the 24 months following treatment with albendazole vs placebo. There were a few significant associations found, specifically a reduced odds of memory loss and/or confusion among those in the albendazole group (both with and without multiple imputation), as well as higher odds of anxiety and/or depression when the missing data were imputed. The decrease in memory loss and/or confusion could be related to the decreased number of generalized seizures previously reported for the albendazole group in this study. ${ }^{14}$

As with all longitudinal studies, missing data were a concern, as this can reduce statistical power and bias results. In an RCT, this can also lead to an imbalance of risk factors that were initially balanced through random assignment to a treatment group. Therefore, a strength of the current analysis is the use of multiple imputation for the logistic regression models to determine how sensitive our findings were to missing data. In most cases, the analysis with imputed data gave similar results to those found with complete case analysis, which were mostly null. In the case of memory loss and/or confusion, the imputed results were consistent with the complete case analyses. Nonetheless, because of the lack of research on predictors of non-seizure symptoms in NC patients, we did not have abundant information about what variables to use to impute data. In addition, we considered data from the entire 24-month period of the trial as well as two different time periods (1-12 months and 13-24 months) to see if treatment effects might be time specific. We found no such pattern.

There are limitations to this study that need to be considered in its interpretation. The symptoms we included in these analyses were those most commonly reported by the patients and may include some related to NC disease as well as some unrelated to the disease but which we asked about, as they might be related to treatment. We expected that this difference in aetiology would become apparent in the analysis, with those associated with NC decreasing more over time in the treatment group, those related to albendazole increasing over time in the treatment group and spurious symptoms unrelated to NC and albendazole not being associated with the treatment group. The fact that we found null associations for most analyses could be because treatment does not impact the clinical profile of NC or because the symptoms we 


\section{K. Thapa et al.}

Table 3. Logistic regression model evaluating the association between albendazole treatment (vs placebo) and the probability of having the symptom during follow-up

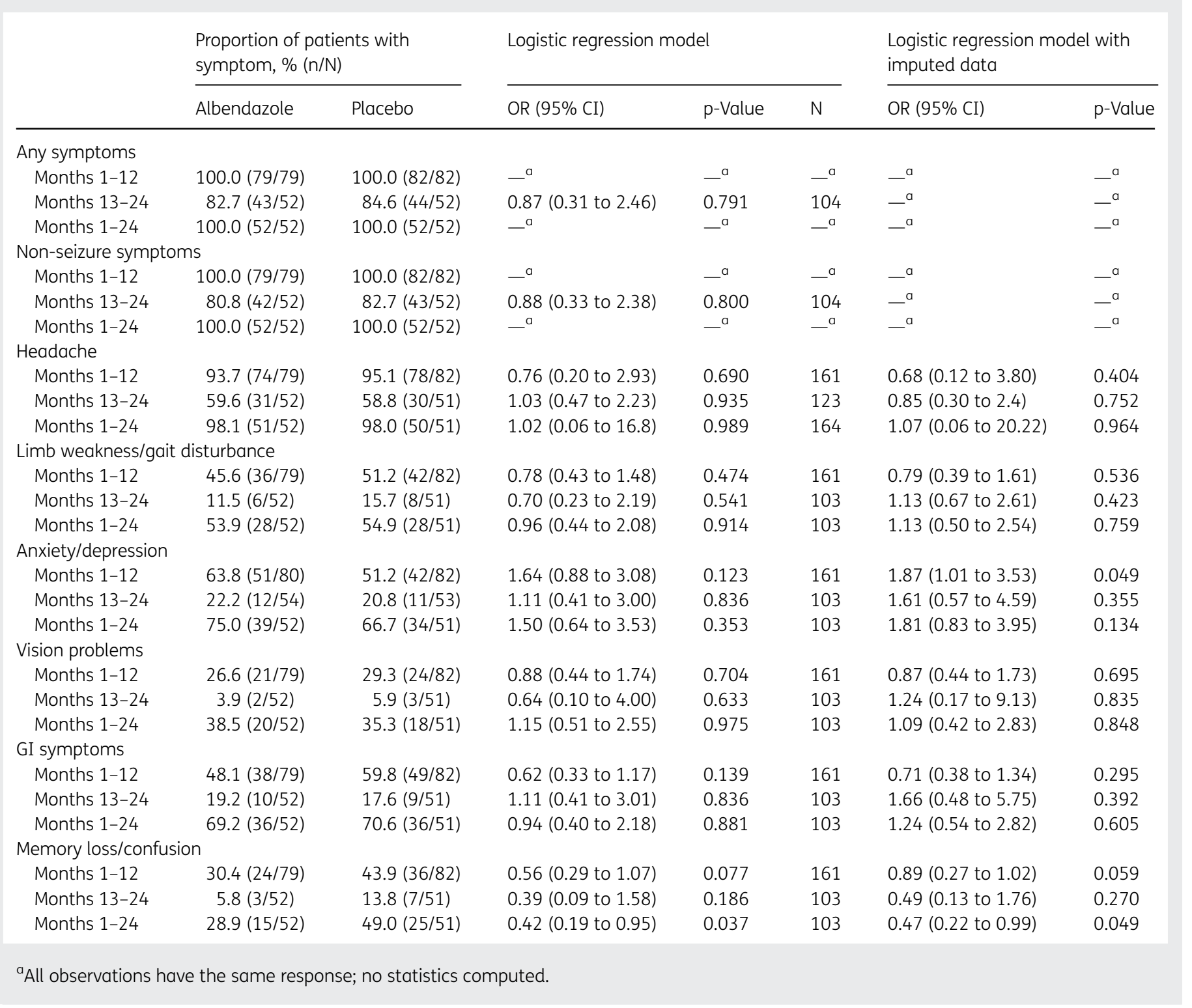

looked at were not related to NC or its treatment. However, it is also possible that these null results are due to a lack of statistical power. This study only included 173 patients and therefore may have had an insufficient sample size to find small associations. Furthermore, while we excluded patients with active tuberculosis, syphilis, papilledema, ocular cysticercosis, active ulcers or any progressive or life-threatening disorder, some of the symptoms that we investigated may have been associated with coexisting medical conditions that were undiagnosed or unmeasured. In addition, symptoms were based on self-reports by patients, which may introduce recall bias and misclassification of the symptom. Because treatment was assigned randomly and the patients and their clinicians were blinded, this misclassification would likely be non-differential by treatment status and thus would likely bias the results towards the null, further reducing statistical power. In addition, loss to follow-up and missing data also have the effect of reducing statistical power and could lead to bias, although we tried to address this through multiple imputation for the logistic regression models. However, multiple imputation assumes that the data are missing at random and it is a possibility that data were not missing at random due to unmeasured factors. Furthermore, we conducted a large number of analyses, so the few significant associations that we found may be type I errors (i.e., rejecting the null hypothesis when it is true). And finally, it is possible that the mostly 
Table 4. Cox proportional hazards models looking at the association between albendazole treatment (vs placebo) and time to symptom during follow-up

\begin{tabular}{|c|c|c|}
\hline & \multicolumn{2}{|c|}{$\begin{array}{l}\text { Cox proportional hazards model } \\
(\mathrm{N}=173)\end{array}$} \\
\hline & HR (95\% CI) & p-Value \\
\hline \multicolumn{3}{|l|}{ Any symptoms } \\
\hline Months 1-12 & $1.00(0.74$ to 1.35$)$ & 1.000 \\
\hline Months $13-24$ & $0.99(0.72$ to 1.35$)$ & 0.925 \\
\hline Months 1-24 & $1.00(0.74$ to 1.35$)$ & 1.000 \\
\hline \multicolumn{3}{|c|}{ Non-seizure symptoms } \\
\hline Months 1-12 & $1.00(0.74$ to 1.35$)$ & 1.000 \\
\hline Months $13-24$ & $0.99(0.72$ to 1.35$)$ & 0.925 \\
\hline Months 1-24 & $1.00(0.74$ to 1.35$)$ & 1.000 \\
\hline \multicolumn{3}{|l|}{ Headache } \\
\hline Months 1-12 & 0.99 (0.73 to 1.43$)$ & 0.935 \\
\hline Months $13-24$ & $1.00(071$ to 1.40$)$ & 0.983 \\
\hline Months 1-24 & $1.00(0.74$ to 1.35$)$ & 1.000 \\
\hline \multicolumn{3}{|c|}{ Limb weakness/gait disturbance } \\
\hline Months 1-12 & $0.93(0.61$ to 1.40$)$ & 0.714 \\
\hline Months $13-24$ & $0.92(0.60$ to 1.41$)$ & 0.702 \\
\hline Months 1-24 & 0.98 (0.69 to 1.39$)$ & 0.910 \\
\hline \multicolumn{3}{|l|}{ Anxiety/depression } \\
\hline Months 1-12 & $1.23(0.83$ to 1.81$)$ & 0.299 \\
\hline Months 13-24 & 0.99 (0.65 to 1.50$)$ & 0.959 \\
\hline Months 1-24 & $1.06(0.76$ to 1.46$)$ & 0.749 \\
\hline \multicolumn{3}{|l|}{ Vision problems } \\
\hline Months 1-12 & 0.98 (0.58 to 1.64$)$ & 0.929 \\
\hline Months $13-24$ & 0.93 (0.59 to 1.47$)$ & 0.767 \\
\hline Months 1-24 & 1.01 (0.69 to 1.48$)$ & 0.952 \\
\hline \multicolumn{3}{|l|}{ GI symptoms } \\
\hline Months 1-12 & 0.84 (0.57 to 1.25$)$ & 0.398 \\
\hline Months $13-24$ & 0.99 (0.65 to 1.50$)$ & 0.959 \\
\hline Months 1-24 & 0.98 (0.71 to 1.37$)$ & 0.921 \\
\hline \multicolumn{3}{|c|}{ Memory loss/confusion } \\
\hline Months 1-12 & 0.77 (0.48 to 1.22$)$ & 0.260 \\
\hline Months 13-24 & 0.87 (0.56 to 1.35$)$ & 0.537 \\
\hline Months 1-24 & 0.81 (0.56 to 1.18$)$ & 0.279 \\
\hline
\end{tabular}

null findings were due, at least in part, to the steroid treatment given to participants in both treatment groups, which may have had the effect of decreasing symptom frequency or severity equally in both groups, although the high frequency of symptoms reported over the follow-up makes this seem unlikely.

Despite these limitations, this is one of the first papers to report on the diversity and frequency of non-seizure symptoms experienced by NC patients over a long-term follow-up period and to assess whether these non-seizure symptoms differ by treatment. Our findings mostly suggest no or small (and undetectable in our sample) differences in the symptomatic profile of NC patients over time by treatment, suggesting that better clinical management of the disease is needed to improve quality of life for these patients. Future research on the impact of treatment on NC symptoms is needed with larger samples of NC patients to determine if our null results are real or due to insufficient power and if our few significant associations are real or type I errors. A meta-analysis combining data across completed treatment trials might be the only way to gain a better understanding of the impact treatment has on NC symptoms, as anthelmintic treatment is now the standard care for NC and additional placebo-controlled trials are unlikely to be conducted.

\section{Conclusions}

While the prevalence of non-seizure symptoms was extremely high in this cohort of patients both before and after treatment, there was little difference in the non-seizure symptoms experienced by NC patients over 24 months in those treated with albendazole vs those treated with placebo. Albendazole treatment was associated with a decrease in the probability of memory loss and/ or confusion and an increase in the probability of anxiety and/or depression, but there was no clear pattern for these associations over time or across different ways of looking at the outcome (presence of symptom, time to symptom or number of follow-up visits at which the symptom was reported). While anthelmintic treatment is the standard of care in treating patients with parenchymal viable NC, the clinical benefits of this treatment are not clear in terms of addressing non-seizure symptoms and further research is needed to identify more effective treatment and management of NC to reduce the burden of clinical symptoms.

Authors' contributions: All authors have contributed significantly to the experimental design, its implementation, analysis and interpretation of the data and/or writing of the manuscript at the draft and any revision stages. All authors have read and approved the final version.

\section{Acknowledgements: None.}

Funding: The ENG trial was supported by the National Institute of Neurological Disorders and Stroke (NINDS) [grant R01-NS39403 (WA Hauser, PI) and Ruth L. Kirschstein National Research Service Award grant 5F31NS051946 (EA Kelvin, PI)]. Glaxo Wellcome/SmithKline Beecham and Acromax supplied active drug and placebo.

\section{Competing interests: None declared.}

Ethical approval: The Institutional Review Board of Columbia University, the Office for Human Research Protection of the National Institutes of Health and the ethics committees at the recruiting hospitals in Ecuador granted approval for this study.

\section{References}

1 Winkler ASR. Landscape analysis: management of neurocysticercosis with an emphasis on low- and middle-income countries. Geneva: World Health Organization, 2015. http://apps.who.int/iris/bitstream/ 10665/152896/1/WHO_HTM_NTD_NZD_2015.05_eng.pdf.

2 Fleury A, Moreno Garcia J, Valdez Aguerrebere P et al. Neurocysticercosis, a persisting health problem in Mexico. PLoS Negl Trop Dis 2010;4(8): 0000805.

3 Carpio A. Neurocysticercosis: an update. Lancet Infect Dis 2002;2 (12):751-62. 


\section{K. Thapa et al.}

4 Wallin MT, Kurtzke JF. Neurocysticercosis in the United States: review of an important emerging infection. Neurology 2004;63(9):1559-64.

5 O'Neal SE, Flecke RH. Hospitalization frequency and charges for neurocysticercosis, United States, 2003-2012. Emerg Infect Dis. 2015;21 (6):696-76.

6 Carabin H, Ndimubanzi PC, Budke CM et al. Clinical manifestations associated with neurocysticercosis: a systematic review. PLoS Neglected Trop Dis 2011;5(5):e1152.

7 Carpio A, Kelvin EA, Bagiella E et al. Effects of albendazole treatment on neurocysticercosis: a randomised controlled trial. J Neurol Neurosurg Psychiatry 2008;79(9):1050-5.

8 Baird RA, Wiebe S, Zunt JR et al. Evidence-based guideline: treatment of parenchymal neurocysticercosis: report of the Guideline Development Subcommittee of the American Academy of Neurology. Neurology 2013;80(15):1424-9.

9 Carpio A, Romo ML. The relationship between neurocysticercosis and epilepsy: an endless debate. Arq Neuropsiquiatr 2014;72(5):383-90.

10 Garcia HH, Pretell EJ, Gilman RH et al. A trial of antiparasitic treatment to reduce the rate of seizures due to cerebral cysticercosis. $N$ Engl J Med 2004;350(3):249-58.

11 Chaurasia RN, Garg RK, Agarwall A et al. Three day albendazole therapy in patients with a solitary cysticercus granuloma: a randomized double blind placebo controlled study. Southeast Asian J Trop Med Public Health 2010;41(3):517-25.

12 Khurana N, Garg RK, Verma R et al. Three-day versus 15-day course of albendazole therapy in solitary cysticercus granuloma: an open label randomized trial. J Neurol Sci 2012;316(1-2):36-41.

13 Otte WM, Singla M, Sander JW et al. Drug therapy for solitary cysticercus granuloma: a systematic review and meta-analysis. Neurology 2013;80(2):152-62.

14 Romo ML, Wyka K, Carpio A et al. The effect of albendazole treatment on seizure outcomes in patients with symptomatic neurocysticercosis. Trans R Soc Trop Med Hyg 2015;109(11):738-46.

15 Garcia HH, Pretell EJ, Gilman RH et al. A trial of antiparasitic treatment to reduce the rate of seizures due to cerebral cysticercosis. $\mathrm{N}$ Engl J Med 2004;350(3):249-58.

16 Das K, Mondal GP, Banerjee $M$ et al. Role of antiparasitic therapy for seizures and resolution of lesions in neurocysticercosis patients: an 8 year randomised study. J Clin Neurosci 2007;14(12):1172-7.

17 Kelvin EA, Carpio A, Bagiella E et al. The association of host age and gender with inflammation around neurocysticercosis cysts. Ann Trop Med Parasitol 2009;103(6):487-99.
18 Kelvin EA, Carpio A, Bagiella E et al. Seizure in people with newly diagnosed active or transitional neurocysticercosis. Seizure 2011;20(2):119-25.

19 Kelvin EA, Carpio A, Hesdorffer DC et al. Investigation of familial aggregation of seizures in neurocysticercosis patients. Epilepsy Res 2009;84(1):67-71.

20 Kelvin EA, Yung J, Fong MW et al. The association of living conditions and lifestyle factors with burden of cysts among neurocysticercosis patients in Ecuador. Trans R Soc Trop Med Hyg 2012;106 (12):763-9.

21 Schumann L, Bales HW. Neurocysticercosis: migration of a parasite. J Am Assoc Nurse Pract 2000;12(6):240-8.

22 Scharf D. Neurocysticercosis: two hundred thirty-eight cases from a California hospital. Arch Neurol 1988;45(7):777-80.

23 Ghasemi R, Rowe A, Shah R et al. Neurocysticercosis presenting as a 'stroke mimic'. Acute Med 2016;15(2):79-83.

24 Shandera WX, White AC Jr, Chen JC et al. Neurocysticercosis in Houston, Texas. A report of 112 cases. Medicine 1994;73(1):37-42.

25 Agapejev S, da Silva MD, Ueda AK. Severe forms of neurocysticercosis: treatment with albendazole. Arq Neuropsiquiatr 1996;54(1):82-3.

26 White AC Jr. Neurocysticercosis: a major cause of neurological disease worldwide. Clin Infect Dis 1997;24(2):101-13; quiz 14-5.

27 de Almeida SM, Gurjao SA. Quality of life assessment in patients with neurocysticercosis. J Community Health 2011;36(4):624-30.

28 Sciutto E, Fragoso G, Fleury A et al. Taenia solium disease in humans and pigs: an ancient parasitosis disease rooted in developing countries and emerging as a major health problem of global dimensions. Microbes Infect 2000;2(15):1875-90.

29 Srivastava S, Chadda RK, Bala K et al. A study of neuropsychiatric manifestations in patients of neurocysticercosis. Indian J Psychiatry 2013;55(3):264-7.

30 Garcia HH, Gilman RH, Horton J et al. Albendazole therapy for neurocysticercosis: a prospective double-blind trial comparing 7 versus 14 days of treatment. Neurology 1997;48(5):1421-7.

31 Ciampi de Andrade D, Rodrigues CL, Abraham R et al. Cognitive impairment and dementia in neurocysticercosis: a cross-sectional controlled study. Neurology 2010;74(16):1288-95.

32 Yuan YC. Multiple imputation for missing data: concepts and new development (version 9.0) Rockville, MD: SAS Institute, 2010.

33 Carpio A, Hauser WA. Prognosis for seizure recurrence in patients with newly diagnosed neurocysticercosis. Neurology 2002;59(11): 1730-4. 